\title{
UNIFORM ASYMPTOTIC STABILITY OF TIME-VARYING DAMPED HARMONIC OSCILLATORS
}

\author{
KAZUKI ISHIHARA AND JITSURO SUGIE \\ (Communicated by Wenxian Shen)
}

Abstract. This paper presents sufficient conditions which guarantee that the equilibrium of the damped harmonic oscillator

$$
x^{\prime \prime}+h(t) x^{\prime}+\omega^{2} x=0
$$

is uniformly asymptotically stable, where $h:[0, \infty) \rightarrow[0, \infty)$ is locally integrable. These conditions work to suppress the rapid growth of the frictional force expressed by the integral amount of the damping coefficient $h$. The obtained sufficient conditions are compared with known conditions for uniform asymptotic stability. Two diagrams are included to facilitate understanding of the conditions. By giving a concrete example, remaining problems are pointed out.

\section{INTRODUCTION}

We consider the second-order linear differential equation

$$
x^{\prime \prime}+h(t) x^{\prime}+\omega^{2} x=0,
$$

where the prime denotes $d / d t$, the coefficient $h$ is a nonnegative and locally integrable function on $[0, \infty)$, and the number $\omega$ is a positive constant. The only equilibrium of (1) is the origin $\left(x, x^{\prime}\right)=(0,0)$. Equation (11) is often called the damped harmonic oscillator when $h$ is a positive constant. Although the damped harmonic oscillator has a very simple form, there are extremely wide applications in science and engineering. Equation (1) has been studied as one of the important physical phenomenon models by many researchers.

In the qualitative theory of differential equations, the study of asymptotic stability and uniform asymptotic stability occupy very important positions. The purpose of this paper is to present some growth condition about $h$ for the equilibrium of (1) to be uniformly asymptotically stable and to clarify the relationship between these conditions. Before advancing to the main subject, it is useful to briefly describe the history of study of the asymptotic stability of (10) and the results obtained.

Received by the editors February 24, 2017, and, in revised form, April 22, 2017 and June 16, 2017.

2010 Mathematics Subject Classification. Primary 34D20, 34D45; Secondary 37C70, 93D20.

Key words and phrases. Uniform asymptotic stability, damped linear oscillator, growth condition.

The second author's work was supported in part by Grant-in-Aid for Scientific Research No. 17K05327 from the Japan Society for the Promotion of Science.

The authors would like to thank an anonymous referee for reading carefully and giving valuable comments.

(C) 2017 by the authors under Creative Commons Attribution 3.0 License (CC BY 3.0) 
Since equation (11) is linear, if the equilibrium is attractive, then it is stable. Hence, we need only show that each solution of (1) and its derivative tend to zero as $t \rightarrow \infty$ in order to prove that the equilibrium is asymptotically stable. Many efforts have been made to find sufficient (also necessary and sufficient) conditions which guarantee that the equilibrium of (11) is asymptotically stable (for example, see 1, 3, $4,9,13,16,19,21,24,27,31$ ). Among them, we should mention especially the criterion given by Smith [21, Theorems 1 and 2]. Let

$$
H(t)=\int_{0}^{t} h(s) d s
$$

Then the following result holds.

Theorem A. Suppose that

$$
\text { there exists an } \underline{h}>0 \text { such that } h(t) \geq \underline{h} \text { for } t \geq 0 \text {. }
$$

Then the equilibrium of (11) is asymptotically stable if and only if

$$
\int_{0}^{\infty} \frac{\int_{0}^{t} e^{H(s)} d s}{e^{H(t)}} d t=\infty .
$$

When $h$ satisfies condition (2), namely, $h$ has a positive lower bound $\underline{h}$, it is often called large damping. Smith's condition (플 is satisfied when $h$ has an upper bound $\bar{h}$ or $h(t)=t$. On the other hand, condition (S) is not satisfied when $h(t)=t^{2}$ (for the proof, see [11]). From these facts, we see that condition (S) prohibits rapid growth of the damping coefficient $h$. Since condition $(\underline{\mathrm{S}})$ is necessary and sufficient for the asymptotic stability of (1), it is not too much to say that condition (S) is very excellent. However, the weak point is that it is hard to check whether condition (S) is satisfied or not. Although Artstein and Infante [1] did not point out this fact, they gave another growth condition that guarantees the asymptotic stability of (1) as follows.

Theorem B. Suppose that condition (2) holds. Then

$$
\limsup _{t \rightarrow \infty} \frac{H(t)}{t^{2}}<\infty
$$

implies that the equilibrium of (1) is asymptotically stable.

Artstein and Infante's condition (A) requires that $H$ has to grow more slowly than $t^{2}$. Artstein and Infante [1] also showed that the exponent 2 of $t$ is best possible in the sense that it cannot be replaced by $2+\varepsilon$ for any $\varepsilon>0$. Of course, condition (A) is not as sharp as condition (S). For example, consider $h(t)=(2+t) \log (2+t)$. Then it is clear that $H(t) / t^{2}$ is unbounded. Hence, condition (A) is not satisfied. However, by means of Ballieu and Peiffer's result [3, Corollary 7], we can verify that the equilibrium of (11) is asymptotically stable in this example.

The advantage of condition (A) is that it is easy to check. When an indefinite integral $H$ of $h$ is found, we may judge whether condition $(\mathrm{A}$ is satisfied or not. By numerical computation, it may be easy to check condition (A). However, it is very hard to confirm condition $(\underline{S})$ even with numerical computation.

Since condition (A) is merely a sufficient condition for the asymptotic stability of (1), it follows from condition (S). From another viewpoint, Hatvani et al. 11] 
verified that condition (A) implies condition (S). They proved that condition (S) is equivalent to the discrete growth condition

$$
\sum_{n=1}^{\infty}\left(H^{-1}(n c)-H^{-1}((n-1) c)\right)^{2}=\infty \quad \text { for any } c>0
$$

where

$$
H^{-1}(s) \stackrel{\text { def }}{=} \min \{t \in[0, \infty): H(t) \geq s\}, \quad s \in[0, \infty),
$$

provided that $H(t)$ diverges to $\infty$ as $t \rightarrow \infty$ (see [11, Theorem 1.1]). They also showed that condition (A) implies condition (D) in the proof of Corollary 3.7 in [1]. (see also [1]). Moreover, they gave another growth condition,

$$
\sum_{i=N}^{\infty} \frac{1}{\int_{i-1}^{i} h(s) d s}=\infty
$$

for any fixed natural number $N$ (their original form is slightly different) and clarified that condition ( $(\mathrm{H})$ implies condition (D) under certain conditions including condition (2) (see [11, Corollary 3.6]). We can show that condition (A) implies condition ( $(\mathrm{H})$ by using Artstein and Infante's result [1, Lemma] (for the proof, see Appendix). To sum up, we have the following diagram.

$$
\begin{aligned}
& \text { (2) } \\
& \downarrow \\
& \text { (A) } \Longrightarrow(\underline{H} \\
& \text { (2) } \longrightarrow \Downarrow \quad \nVdash \longleftarrow \lim _{t \rightarrow \infty} x^{\prime}(t)=0 \text {, certain conditions } \\
& (\mathrm{D}) \Longleftrightarrow(\mathrm{S}) \Longleftrightarrow[\mathrm{AS}] \\
& \uparrow \uparrow \uparrow \\
& \lim _{t \rightarrow \infty} H(t)=\infty \Longleftarrow(2)
\end{aligned}
$$

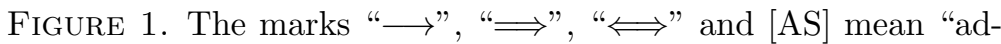
dition to", "implies", "if and only if" and the asymptotic stability of (11), respectively.

The equilibrium of (1) is said to be asymptotically stable [AS] if

$$
\lim _{t \rightarrow \infty} x(t)=\lim _{t \rightarrow \infty} x^{\prime}(t)=0
$$

for every solution $x$ of (1). As is well known, the equilibrium is not necessarily uniformly asymptotically stable even if it is [AS]. We need to check that each solution of (11) and its derivative converge to zero with the speed of the same level in order to prove that the equilibrium is uniformly asymptotically stable. In this sense, we need to pay close attention to the analysis of uniform asymptotic stability.

To strictly describe definitions, we give some notation. Let $\mathbf{x}(t)=\left(x(t), x^{\prime}(t)\right)$ and $\mathbf{x}_{0} \in \mathbb{R}^{2}$, and let $\|\cdot\|$ be any suitable norm. We denote the solution of (1) through $\left(t_{0}, \mathbf{x}_{0}\right)$ by $\mathbf{x}\left(t ; t_{0}, \mathbf{x}_{0}\right)$. The uniqueness of solutions of (1) is guaranteed for the initial value problem.

The equilibrium is said to be eventually uniformly stable [EvUS] if for any $\varepsilon>0$, there exist an $\alpha(\varepsilon) \geq 0$ and a $\delta(\varepsilon)>0$ such that $\left\|\mathbf{x}_{0}\right\|<\delta$ and $t_{0} \geq \alpha$ imply that $\left\|\mathbf{x}\left(t ; t_{0}, \mathbf{x}_{0}\right)\right\|<\varepsilon$ for all $t \geq t_{0}$. If we can choose $\alpha(\varepsilon)=0$, the equilibrium is said to be uniformly stable [US]. The equilibrium is said to be eventually uniformly 
attractive [EvUA] if there exist an $\alpha_{0} \geq 0$ and a $\delta_{0}>0$, and if for every $\eta>0$ there is a $T(\eta)>0$ such that $t_{0} \geq \alpha_{0}$ and $\left\|\mathbf{x}_{0}\right\|<\delta_{0}$ imply that $\left\|\mathbf{x}\left(t ; t_{0}, \mathbf{x}_{0}\right)\right\|<\eta$ for all $t \geq$ $t_{0}+T(\eta)$. If we can choose $\alpha_{0}=0$, the equilibrium is said to be uniformly attractive [UA]. The equilibrium is eventually uniformly asymptotically stable [EvUAS] if it is [EvUS] and [EvUA]. The equilibrium is uniformly asymptotically stable [UAS] if it is [US] and [UA]. With respect to the various definitions of stability, the reader may refer to the books $[2,5,6,15,20,32$ for example.

It is well known that the equilibrium of (11) is uniformly asymptotically stable if and only if it is exponentially asymptotically stable [ExpAS]; namely, there exists a $\kappa>0$ and, for any $\varepsilon>0$, there exists a $\delta(\varepsilon)>0$ such that $t_{0} \geq 0$ and $\left\|\mathbf{x}_{0}\right\|<\delta(\varepsilon)$ imply that $\left\|\mathbf{x}\left(t ; t_{0}, \mathbf{x}_{0}\right)\right\|<\varepsilon \exp \left(-\kappa\left(t-t_{0}\right)\right)$ for all $t \geq t_{0}$. If the equilibrium of (1) is [ExpAS], then the existence of a good Lyapunov function $V(\cdot, \cdot):[0, \infty) \times \mathbb{R}^{n} \rightarrow \mathbb{R}$ that satisfies the following conditions is guaranteed:

(i) $a(\|\mathbf{x}\|) \leq V(t, \mathbf{x}) \leq b(\|\mathbf{x}\|)$,

(ii) $\dot{V}_{\text {[1] }}(t, \mathbf{x}) \leq-c(\|\mathbf{x}\|) \quad$ or $\quad \dot{V}_{\text {[1] }}(t, \mathbf{x}) \leq-d V(t, \mathbf{x})$,

(iii) $\left|V\left(t, \mathbf{x}_{1}\right)-V\left(t, \mathbf{x}_{2}\right)\right| \leq f(t)\left\|\mathbf{x}_{1}-\mathbf{x}_{2}\right\|$

on $[0, \infty) \times \mathbb{R}^{n}$, where $a, b$ and $c$ are continuous increasing and positive definite functions, $d$ is a positive constant and $f$ is a positive suitable function (this is called a converse theorem on [UAS]). However, if the zero solution of (11) is merely only asymptotically stable, such a good Lyapunov function does not necessarily exist. This is a big difference between [UAS] and [AS]. By using the converse theorem on [UAS], we can show that the uniform asymptotic stability is maintained even if a small perturbation term is added to equation (11). Small errors cannot be ignored in model design. For this reason, it is necessary to consider the perturbation problem in actual phenomena analysis. From this point of view, the study of [UAS] is very important.

In this paper, we use the terminology "uniformly with respect to $\sigma \geq 0$ ". This means the following: Let $f_{\sigma}: \mathbb{R} \rightarrow \mathbb{R}$ be a family of functions parametrized by $\sigma \geq 0$. We say that $\lim _{t \rightarrow \infty} f_{\sigma}(t)=\infty$ uniformly with respect to $\sigma \geq 0$ if, and only if, for any $M>0$ there exists a $T \geq 0$ such that $\sigma \geq 0$ and $t \geq T$ imply $f_{\sigma}(t) \geq M$. We also use the symbol $[c]$ to mean the greatest integer that is less than or equal to a real number $c$.

Sugie and Onitsuka [30, Theorem 1.1] gave the following result.

Theorem C. Suppose that

$$
\liminf _{t \rightarrow \infty} \int_{t}^{t+d} h(s) d s>0 \quad \text { for every } d>0 .
$$

If

$$
\lim _{t \rightarrow \infty} \int_{\sigma}^{t+\sigma} \frac{\int_{\sigma}^{s} e^{H(\tau)} d \tau}{e^{H(s)}} d s=\infty \quad \text { uniformly with respect to } \sigma \geq 0
$$

then the equilibrium of (11) is uniformly asymptotically stable.

When condition (3) holds, the damping coefficient $h$ is said to be integrally positive. The concept of the integral positivity was introduced by Matrosov 14 . (see also [7, 9, 17, 24, 28, 29]). It is obvious that condition (2) implies condition (3). However, the converse is not always true. Integrally positive functions are allowed to 
have an infinite number of zeros. A typical example of integrally positive functions is $\sin ^{2} t$.

Let us compare condition (44) with condition (SS). We notice that both are double integrals of $\exp (H(\tau)-H(s))$. Condition (4) requires that this double integral diverges uniformly with respect to $\sigma$. On the other hand, condition ( $(\underline{S})$ only needs to diverge when $\sigma=0$. Hence, we may say that (4) is a uniform divergence condition. A growth condition similar to condition (44) was first presented by Hatvani [9, Theorem 2.5] as a sufficient condition for the zero solution of a certain two-dimensional linear system to become asymptotically stable.

By the same method as in the proof of Theorem C, we can obtain the following result.

Theorem D. Suppose that condition (3) holds. If

$$
\text { there exists an } m \geq 0 \text { such that } \lim _{t \rightarrow \infty} \int_{\sigma}^{t+\sigma} \frac{\int_{\sigma}^{s} e^{H(\tau)} d \tau}{e^{H(s)}} d s=\infty
$$

uniformly with respect to $\sigma \geq m$,

then the equilibrium of (1) is eventually uniformly asymptotically stable.

For nonlinear differential equations, the concept of [EvUAS] is different from that of [UAS]. Of course, the equilibrium is uniformly asymptotically stable, so it is eventually uniformly asymptotically stable; namely, [UAS] implies [EvUAS]. Strauss and Yorke [22, Lemma 2.7] gave a necessary and sufficient condition for the converse to be true as follows (see also [23]).

Theorem E. Suppose that the equilibrium is eventually uniformly asymptotically stable. Then it is uniformly asymptotically stable if and only if the zero function is a unique solution defined on the interval $\left[t_{0}, \infty\right)$.

Since equation (1) is linear, from TheoremEit turns out that [EvUAS] is equivalent to [UAS]. Hence, condition (SU) is a growth condition on uniform asymptotic stability. In this paper, based on the uniform divergence condition (SU), we intend to present other growth conditions on uniform asymptotic stability and give a correlation diagram showing their relation.

\section{Conditions For SUPPRESSing THE RAPID GROWTH OF $h$}

As mentioned in Section 1, Hatvani et al. [1] presented the discrete growth condition for the asymptotic stability of (11), which is equivalent to Smith's condition (S). Inspired by this result, Sugie and Onitsuka [30, Theorem 4.2] gave the discrete growth condition

(5) $\lim _{n \rightarrow \infty} \sum_{i=N}^{n+N}\left(H^{-1}(i)-H^{-1}(i-1)\right)^{2}=\infty \quad$ uniformly with respect to $N \in \mathbb{N}$,

for uniform asymptotic stability of (10) and proved that condition (5) implies the uniform divergence condition (4) under the assumption (3). Using the same idea, we can show that

there exists an $N^{*} \in \mathbb{N}$ such that $\lim _{n \rightarrow \infty} \sum_{i=N}^{n+N}\left(H^{-1}(i)-H^{-1}(i-1)\right)^{2}=\infty$ uniformly with respect to $N \geq N^{*}$ 
implies condition (SU). We may regard condition (DU) as a discrete version of (D).

Unfortunately, in general, it is not so easy to check whether a given $h$ satisfies conditions (SU) and (DU). In this section, we propose other growth conditions corresponding to conditions $(\mathrm{A})$ and $(\mathrm{H})$ given by Artstein and Infante 1 and Hatvani et al. [11, respectively. We also reveal implications between conditions (SU), (DU) and these new growth conditions.

Theorem 1. Suppose that $\lim _{t \rightarrow \infty} H(t)=\infty$. If

there exists an $\varepsilon_{0}>0$ and an $m \geq 0$ such that

$$
\limsup _{t \rightarrow \infty} \frac{1}{t^{2-\varepsilon_{0}}} \int_{\tau}^{t+\tau} h(s) d s<\infty \text { uniformly with respect to } \tau \geq m
$$

then condition (DU) holds.

Remark 1. If condition (3) is satisfied, then $\lim _{t \rightarrow \infty} H(t)=\infty$. Condition (AU) is a uniform convergence version of condition (A) of Artstein and Infante [1].

Theorem 2. Suppose that

(6) there exists an $N \in \mathbb{N}$ such that $a_{n} \stackrel{\text { def }}{=} \int_{n-1}^{n} h(s) d s>0$ for all $n \geq N$.

If condition (AU] is satisfied, then

there exists an $N^{*} \in \mathbb{N}$ such that $\lim _{n \rightarrow \infty} \sum_{i=N}^{n+N} \frac{1}{\int_{i-1}^{i} h(s) d s}=\infty$
uniformly with respect to $N \geq N^{*}$.

Remark 2. Condition (3) implies condition (6). Condition (HU) is a uniform divergence version of condition $(\mathrm{H})$ of Hatvani et al. [11.

Theorem 3. Suppose that

there exists a $T>0$ such that $1 / h$ is a bounded function on $[T, \infty)$

and $(1 / h)^{\prime}$ is a function on $[T, \infty)$ that is bounded from above.

Then condition ( $\mathrm{HU}$ ) yields condition ( $(\mathrm{SU})$.

Combining Theorems 12 and 3 with Theorems DandE, we can give the diagram for [UAS] shown in Figure 2:

$$
\begin{aligned}
& \text { (3) } \Longrightarrow(6)
\end{aligned}
$$

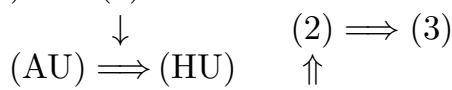

$$
\begin{aligned}
& \lim _{t \rightarrow \infty} H(t)=\infty \longrightarrow \Downarrow \quad \Downarrow \longleftarrow \text { (7) } \\
& \Uparrow \quad \text { (13) }
\end{aligned}
$$

(3) (3)

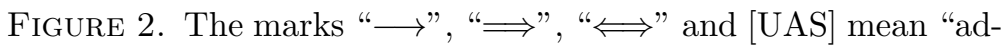
dition to", "implies", "if and only if" and the uniform asymptotic stability of (1), respectively. 


\section{Proofs}

Proof of Theorem 1. Let $t_{0}=0$ and $t_{n}=H^{-1}(n)$ for all $n \in \mathbb{N}$. Since $\lim _{t \rightarrow \infty} H(t)$ $=\infty$, the sequence $\left\{t_{n}\right\}$ is increasing and diverges to $\infty$ as $n \rightarrow \infty$. Hence, we can choose an $N^{*} \in \mathbb{N}$ so that

$$
t_{N^{*}-1} \geq m \text {. }
$$

Define $\Delta t_{n}=t_{n}-t_{n-1}$. In order to show condition (DU), it suffices to show that for any $L>0$ there exists an $M(L) \in \mathbb{N}$ such that $n \geq M$ implies that

$$
\sum_{i=N}^{n+N}\left(\Delta t_{i}\right)^{2}>L
$$

for any $N \geq N^{*}$. From (AU) it follows that

For any $L>0$, let

$$
\text { there exists a } K>0 \text { and a } T>0 \text { such that } \tau \geq m
$$

$$
M(L)=\max \left\{1,\left[K T^{2-\varepsilon_{0}}\right],\left[K^{2 / \varepsilon_{0}} L^{\left(2-\varepsilon_{0}\right) / \varepsilon_{0}}\right]\right\} \in \mathbb{N} .
$$

Suppose that there exists an $N_{0} \in \mathbb{N}$ with $N_{0} \geq N^{*}$ such that

$$
t_{N_{0}+M}<t_{N_{0}-1}+T \text {. }
$$

Then, since $H$ is an increasing function on $[0, \infty)$ and $H\left(t_{n}\right)=n$ for all $n \in \mathbb{N}$, we see that

$$
\begin{aligned}
N_{0}+M & =H\left(t_{N_{0}+M}\right) \leq H\left(t_{N_{0}-1}+T\right) \\
& =H\left(t_{N_{0}-1}\right)+\int_{t_{N_{0}-1}}^{t_{N_{0}-1}+T} h(s) d s \\
& =N_{0}-1+\int_{t_{N_{0}-1}}^{t_{N_{0}-1}+T} h(s) d s .
\end{aligned}
$$

Since $\left\{t_{n}\right\}$ is an increasing sequence, we see that $t_{N_{0}-1} \geq t_{N^{*}-1} \geq m$. Using (8) with $\tau=t_{N_{0}-1}$ and $t=T$, we obtain

$$
\int_{t_{N_{0}-1}}^{t_{N_{0}-1}+T} h(s) d s<K T^{2-\varepsilon_{0}}
$$

Hence, we have

$$
M<K T^{2-\varepsilon_{0}}-1 .
$$

This contradicts (91). We therefore conclude that

$$
t_{N+M} \geq t_{N-1}+T \text { for any } N \geq N^{*} .
$$

We can rewrite (8) as

$$
\int_{\tau}^{t} h(s) d s<K(t-\tau)^{2-\varepsilon_{0}} \text { for } t \geq \tau+T .
$$

Using (10) and (11), we get

$$
\int_{t_{N-1}}^{t_{N+M}} h(s) d s<K\left(t_{N+M}-t_{N-1}\right)^{2-\varepsilon_{0}} \text { for any } N \geq N^{*} .
$$


Hence, we have

$$
\begin{aligned}
\frac{M+1}{\left(t_{N+M}-t_{N-1}\right)^{2-\varepsilon_{0}}} & =\frac{H\left(t_{N+M}\right)-H\left(t_{N-1}\right)}{\left(t_{N+M}-t_{N-1}\right)^{2-\varepsilon_{0}}} \\
& =\frac{1}{\left(t_{N+M}-t_{N-1}\right)^{2-\varepsilon_{0}}} \int_{t_{N-1}}^{t_{N+M}} h(s) d s<K ;
\end{aligned}
$$

that is,

$$
\left(t_{N+M}-t_{N-1}\right)^{2-\varepsilon_{0}}>\frac{M+1}{K} \text { for any } N \geq N^{*} .
$$

By means of the Cauchy-Bunyakovski-Schwarz inequality, we have

$$
\left(\sum_{i=N}^{M+N} \Delta t_{i}\right)^{2} \leq \sum_{i=N}^{M+N} 1^{2} \sum_{i=N}^{M+N}\left(\Delta t_{i}\right)^{2}=(M+1) \sum_{i=N}^{M+N}\left(\Delta t_{i}\right)^{2}
$$

for any $N \geq N^{*}$. Hence, it follows from (12) that $n \geq N$ implies that

$$
\begin{aligned}
\sum_{i=N}^{n+N}\left(\Delta t_{i}\right)^{2} & \geq \sum_{i=N}^{M+N}\left(\Delta t_{i}\right)^{2} \geq \frac{1}{M+1}\left(\sum_{i=N}^{M+N} \Delta t_{i}\right)^{2} \\
& =\frac{1}{M+1}\left(\left(t_{N+M}-t_{N-1}\right)^{2-\varepsilon_{0}}\right)^{2 /\left(2-\varepsilon_{0}\right)} \\
& >\frac{1}{M+1}\left(\frac{M+1}{K}\right)^{2 /\left(2-\varepsilon_{0}\right)}=\frac{(M+1)^{\varepsilon_{0} /\left(2-\varepsilon_{0}\right)}}{K^{2 /\left(2-\varepsilon_{0}\right)}}
\end{aligned}
$$

for any $N \geq N^{*}$. From (9) it turns out that

$$
M>K^{2 / \varepsilon_{0}} L^{\left(2-\varepsilon_{0}\right) / \varepsilon_{0}}-1 .
$$

Hence, we obtain

$$
\sum_{i=N}^{n+N}\left(\Delta t_{i}\right)^{2}>L
$$

which is our desired estimate. This completes the proof.

Proof of Theorem 2. Note that condition (8) holds as in the proof of Theorem 1. For any $L>0$, let

$$
M(L)=\max \left\{1,[T],\left[(K L)^{1 / \varepsilon_{0}}\right]\right\} \in \mathbb{N} .
$$

From (6), we can find an $N_{*} \in \mathbb{N}$ so that $a_{n}>0$ for $n \geq N_{*}$. Hence, we have

$$
(M+1)^{2}=\left(\sum_{i=N}^{M+N} 1\right)^{2}=\left(\sum_{i=N}^{M+N} \sqrt{a_{i}} \frac{1}{\sqrt{a_{i}}}\right)^{2}
$$

for any $N \geq N_{*}$. Using the Cauchy-Bunyakovski-Schwarz inequality, we obtain

$$
\left(\sum_{i=N}^{M+N} \sqrt{a_{i}} \frac{1}{\sqrt{a_{i}}}\right)^{2} \leq \sum_{i=N}^{M+N} a_{i} \sum_{i=N}^{M+N} \frac{1}{a_{i}} \text { for any } N \geq N_{*} .
$$

Let

$$
N^{*}=\max \left\{m+1, N_{*}\right\}
$$


From (13) it turns out that $M>T-1$. It is obvious that $N-1 \geq N^{*}-1 \geq m$ for $N \geq N^{*}$. Hence, we can use inequality (8) with $t=M+1>T$ and $\tau=N-1$ for any $N \geq N^{*}$ and get

$$
\sum_{i=N}^{M+N} a_{i}=\int_{N-1}^{M+N} h(s) d s<K(M+1)^{2-\varepsilon_{0}}
$$

for $N \geq N^{*}$. We therefore conclude that

$$
\sum_{i=N}^{M+N} \frac{1}{a_{i}}>\frac{1}{K}(M+1)^{\varepsilon_{0}} \text { for any } N \geq N^{*} .
$$

Using (13) again, we obtain

$$
\sum_{i=N}^{n+N} \frac{1}{\int_{i-1}^{i} h(s) d s} \geq \sum_{i=N}^{M+N} \frac{1}{a_{i}}>\frac{1}{K}(M+1)^{\varepsilon_{0}}>L
$$

for any $n \geq M$ and $N \geq N^{*}$, namely, condition ( $\mathrm{HU}$ ).

By using the same method of Sugie and Onitsuka [30, Theorem 3.1], we can prove the following result (we omit the proof).

Lemma 4. Suppose that there exists a function $k:[0, \infty) \rightarrow[0, \infty)$ and a $T>0$ such that

$$
\left\{\begin{array}{l}
0 \leq h(t) \leq k(t) \text { for } t \geq T \\
\frac{1}{k} \text { is a bounded function on }[T, \infty), \text { and } \\
\left(\frac{1}{k}\right)^{\prime} \text { is a function on }[T, \infty) \text { that is bounded from above. }
\end{array}\right.
$$

If

there exists an $m \geq 0$ such that $\lim _{t \rightarrow \infty} \int_{\sigma}^{t+\sigma} \frac{1}{k(s)} d s=\infty$ uniformly with respect to $\sigma \geq m$.

then condition (SU) holds.

Remark 3. As can be seen from the condition (13), the damping coefficient $h$ need not even be differentiable.

Proof of Theorem 3. In order to prove Theorem 3. it suffices, in view of Lemma 4. to show that

there exists an $m \geq 0$ such that $\lim _{t \rightarrow \infty} \int_{\sigma}^{t+\sigma} \frac{1}{h(s)} d s=\infty$

uniformly with respect to $\sigma \geq m$.

From (7), we can find a $c>0$ and $T^{*}>0$ such that

$$
\frac{1}{h(t)} \leq c \text { for } t \geq T^{*}
$$


Hence, we see that $h(t) \geq 1 / c>0$ for all $t \geq T^{*}$. Let $i^{*}=\left[T^{*}\right]+2 \in \mathbb{N}$. Then, by the Cauchy-Bunyakovski-Schwarz inequality, we obtain

$$
1=\left(\int_{i-1}^{i} d s\right)^{2}=\left(\int_{i-1}^{i} \sqrt{h(s)} \frac{1}{\sqrt{h(s)}} d s\right)^{2} \leq a_{i} \int_{i-1}^{i} \frac{1}{h(s)} d s,
$$

where $\left\{a_{i}\right\}$ is the sequence given in Theorem 2, Hence, we have

$$
\int_{i-1}^{i} \frac{1}{h(s)} d s \geq \frac{1}{a_{i}} \text { for all } i \geq i^{*}
$$

Let $N^{* *}=\max \left\{N^{*}, i^{*}\right\}$. Then, from condition (HU) it turns out that

for any $L>0$ there exists an $M(L) \in \mathbb{N}$ such that $N \geq N^{* *}$ implies that

$$
\int_{N-1}^{M+N} \frac{1}{h(s)} d s=\sum_{i=N}^{M+N} \int_{i-1}^{i} \frac{1}{h(s)} d s \geq \sum_{i=N}^{M+N} \frac{1}{a_{i}}>L .
$$

Let $m=N^{* *}-1$. Then, for any $\sigma \geq m$, there exists an $N \in \mathbb{N}$ so that

$$
N-2<\sigma \leq N-1 \text {. }
$$

It is clear that $N \geq N^{* *}$. Let $T(L)=M(L)+2$. Then, using (15), we get

$$
\int_{\sigma}^{t+\sigma} \frac{1}{h(s)} d s \geq \int_{N-1}^{T+N-2} \frac{1}{h(s)} d s=\int_{N-1}^{M+N} \frac{1}{h(s)} d s>L
$$

for $t \geq T$. Hence, condition (14) is satisfied.

\section{Discussion}

As shown in Figure 2, condition (AU) is the most concise condition which guarantees that the equilibrium of (1) is uniformly asymptotically stable. By contrast, the condition that is harder to check is condition ( $(\underline{\mathrm{SU}})$, which includes other conditions. In particular, it is difficult to judge whether the divergence of the double integral in condition (SU) is uniform with respect to $\sigma$ even if it diverges to $\infty$. We give a simple example to show this situation.

Example 1. Consider equation (1) with

$$
h(t)= \begin{cases}1+n & \text { if } n-1 / n \leq t \leq n \\ 1 & \text { if } n<t<n+1-1 /(n+1)\end{cases}
$$

for each $n \in \mathbb{N}$. Then the equilibrium is uniformly asymptotically stable.

It is clear that condition (2) is satisfied with $\underline{h}=1$. Hence, condition (3) is also satisfied. We can easily calculate the integral $H$ as follows:

$$
s=H(t)= \begin{cases}(1+n) t-n(n-1) & \text { if } n-1 / n \leq t \leq n \\ t+n & \text { if } n<t<n+1-1 /(n+1)\end{cases}
$$

for each $n \in \mathbb{N}$ (see Figure 3). Hence, $H$ is a strictly increasing function on $[0, \infty$ ) and $\lim _{t \rightarrow \infty} H(t)=\infty$. Since

for any $\tau \geq 0$ and any $t \geq 0$, there exists an $n \in \mathbb{N}$ and an $m \in \mathbb{N}$ such that $n-1 \leq \tau<n$ and $m-1 \leq t<m$, 

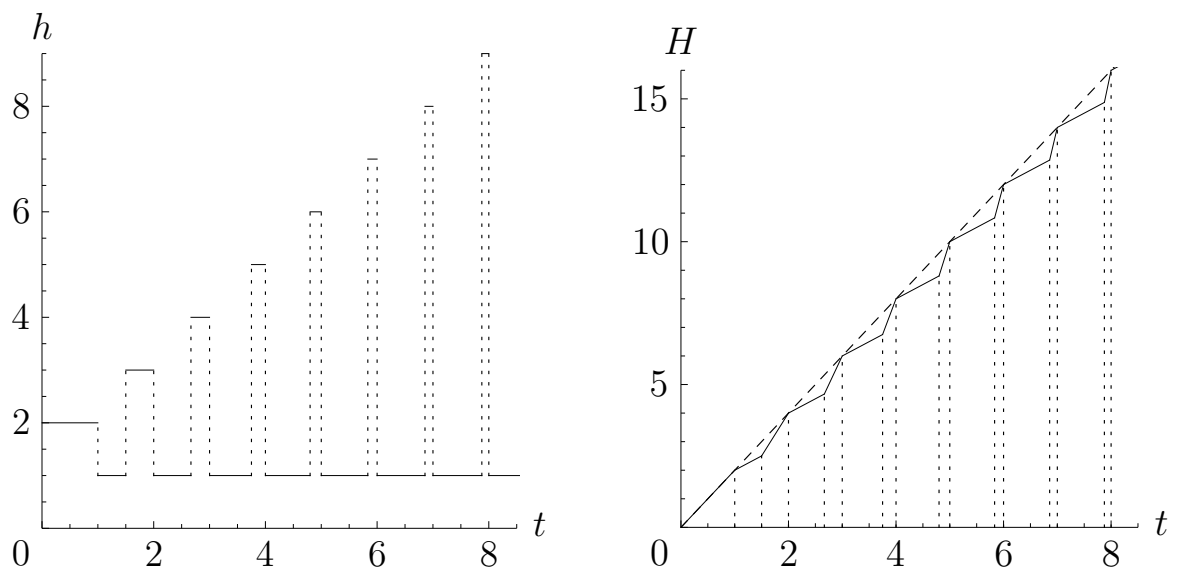

Figure 3. The graphs of the functions $h$ and $H$

we see that

$$
\begin{aligned}
\int_{\tau}^{t+\tau} h(s) d s & =H(t+\tau)-H(\tau)<H(m+n)-H(n-1) \\
& =2(m+n)-2(n-1)=2(m+1)<2(t+2) .
\end{aligned}
$$

Let $\varepsilon_{0}=1$ and $m=0$. Then, we see that $\tau \geq m$ implies that

$$
\frac{1}{t^{2-\varepsilon_{0}}} \int_{\tau}^{t+\tau} h(s) d s<\frac{2(t+2)}{t} \leq 3 \text { for } t \geq 4 .
$$

This means that condition (AU) holds. Hence, from the diagram for [UAS] shown in Figure 2, we see that the equilibrum is uniformly asymptotically stable.

Because condition (AU) is satisfied, conditions (DU), (IU) and (SU) are also satisfied as can be seen from the diagram for [UAS]. In this example, we can directly verify that conditions (DU) and ( $(\mathrm{HU})$ hold. However, it would be difficult to show that condition (SU) is satisfied.

Since $H$ is a strictly increasing function diverging to $\infty$, the function $H^{-1}$ is the inverse function of $H$. We can obtain the inverse function $H^{-1}$ by a straightforward calculation as follows:

$$
t=H^{-1}(s)= \begin{cases}\frac{s+n(n-1)}{1+n} & \text { if } 2 n-1-1 / n \leq s \leq 2 n, \\ s-n & \text { if } 2 n<s<2 n+1-1 /(n+1)\end{cases}
$$

for each $n \in \mathbb{N}$ (see Figure 4). Hence, we see that

$$
\begin{gathered}
H^{-1}(2 n)=\frac{2 n+n(n-1)}{1+n}=n ; \\
H^{-1}(2 n-1)=\frac{2 n-1+n(n-1)}{1+n}=n-\frac{1}{1+n} .
\end{gathered}
$$

For any $N \in \mathbb{N}$ and $n \in \mathbb{N}$, let

$$
P=\left[\frac{N+3}{2}\right] \in \mathbb{N} \quad \text { and } \quad p=\left[\frac{n}{2}\right]-1 .
$$




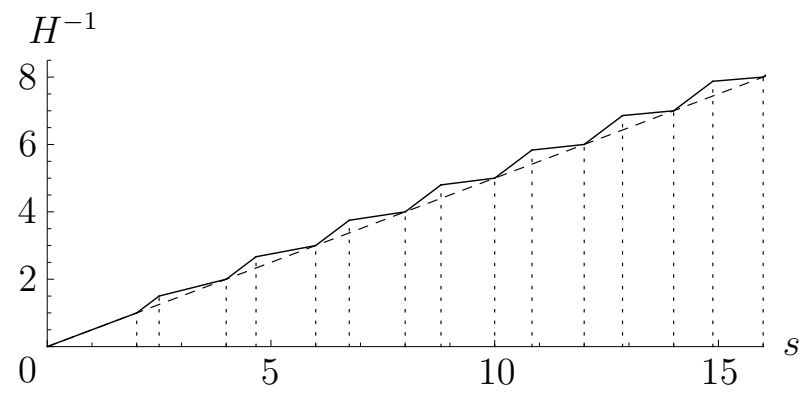

FiguRE 4. The graph of the inverse function $H^{-1}$ of $H$

Then it follows that $2 P-3 \leq N<2 P-1$ and $2(p+P)-1 \leq n+N$. Hence, we have

$$
\begin{aligned}
\sum_{i=N}^{n+N}\left(H^{-1}(i)-H^{-1}(i-1)\right)^{2} & >\sum_{i=2 P-1}^{2(p+P)-1}\left(H^{-1}(i)-H^{-1}(i-1)\right)^{2} \\
& =\sum_{j=P}^{p+P}\left(H^{-1}(2 j-1)-H^{-1}(2 j-2)\right)^{2} .
\end{aligned}
$$

It turns out from (16) that

$$
\begin{aligned}
\sum_{j=P}^{p+P}\left(H^{-1}(2 j-1)-H^{-1}(2 j-2)\right)^{2} & =\sum_{j=P}^{p+P}\left(j-\frac{1}{1+j}-(j-1)\right)^{2} \\
& =\sum_{j=P}^{p+P}\left(\frac{j}{1+j}\right)^{2} \geq \sum_{j=P}^{p+P} \frac{1}{4}=\frac{1}{4}(p+1) \\
& =\frac{1}{4}\left[\frac{n}{2}\right]>\frac{n-2}{8} .
\end{aligned}
$$

We therefore conclude that for any $L>0$, there exists an $M(L)=8 L+2$ such that $n \geq M$ implies that

$$
\sum_{i=N}^{n+N}\left(H^{-1}(i)-H^{-1}(i-1)\right)^{2}>\frac{n-2}{8}>\frac{M-2}{8}=L ;
$$

that is, condition (DU) is satisfied with $N^{*}=1$.

Recall that

$$
a_{n}=\int_{n-1}^{n} h(s) d s .
$$

In this example, it is clear that $a_{n}=2$ for all $n \in \mathbb{N}$. Hence, we obtain

$$
\sum_{i=N}^{n+N} \frac{1}{\int_{i-1}^{i} h(s) d s}=\frac{n+1}{2} .
$$

This means that condition ( $(\mathrm{HU})$ is satisfied with $N^{*}=1$. Note that the damping coefficient $h$ is piecewise continuous but not continuous. Since the differentiability of $h$ is necessary to apply Theorem 3, we cannot show that the equilibrium is uniformly 
asymptotically stable in Example 1 only by satisfying the condition (HU). Hence, there is room for improvement in the assumption of Theorem 3 .

\section{APPENDIX}

We can improve the diagram for [AS] given in Section 1 as follows (compare with Figure 1):

$$
\begin{aligned}
& \text { (3) } \\
& \downarrow \\
& \text { (A) } \Longrightarrow(\text { H } \\
& \text { (3) } \longrightarrow \Downarrow \quad \nVdash \longleftarrow(3) \Longleftarrow \text { (2) } \\
& \text { (D) } \Longleftrightarrow(\text { S }) \Longleftrightarrow[\mathrm{AS}] \\
& \uparrow \uparrow \uparrow \\
& \lim _{t \rightarrow \infty} H(t)=\infty \Longleftarrow \text { (3) }
\end{aligned}
$$

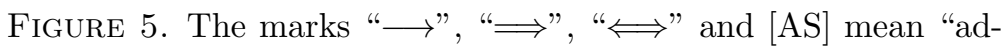
dition to", "implies", "if and only if" and the asymptotic stability of (1), respectively.

Because of limitations of space, we prove only the following relationship here.

Proposition 5. Suppose that condition (3) holds. Then condition (A) implies condition $(\underline{\mathrm{H}})$.

To prove Proposition 5, we need the following lemma, which is obtained by using an idea of Artstein and Infante [1].

Lemma 6. Let $\left\{a_{n}\right\}$ be a sequence. If

$$
\begin{aligned}
& \text { there exist } a K>0 \text { and an } m \in \mathbb{N} \text { such that } \\
& a_{n}>0 \text { for } n \geq m \text { and } \sum_{i=m}^{\ell} a_{i} \leq K \ell^{2} \text { for } \ell \geq m,
\end{aligned}
$$

then

$$
\sum_{i=m}^{\infty} \frac{1}{a_{i}}=\infty
$$

Proof. For any fixed integer $n \geq m$, let $b_{j}=a_{2^{n}+j}>0$ with $j=m, \ldots, 2^{n}$. Then, by assumption, we have

$$
\sum_{j=m}^{2^{n}} b_{j}=\sum_{j=m}^{2^{n}} a_{2^{n}+j}=\sum_{i=2^{n}+m}^{2^{n+1}} a_{i}<\sum_{i=m}^{2^{n}+m-1} a_{i}+\sum_{i=2^{n}+m}^{2^{n+1}} a_{i}=\sum_{i=m}^{2^{n+1}} a_{i} \leq 2^{2(n+1)} K .
$$

Hence, it follows from the Cauchy-Bunyakovski-Schwarz inequality that

$$
\left(2^{n}-m+1\right)^{2}=\left(\sum_{j=m}^{2^{n}} \sqrt{b_{j}} \frac{1}{\sqrt{b_{j}}}\right)^{2} \leq \sum_{j=m}^{2^{n}} b_{j} \sum_{j=m}^{2^{n}} \frac{1}{b_{j}}<2^{2(n+1)} K \sum_{j=m}^{2^{n}} \frac{1}{b_{j}} .
$$

Thus, we obtain

$$
\sum_{j=m}^{2^{n}} \frac{1}{b_{j}}>\frac{\left(2^{n}-m+1\right)^{2}}{2^{2(n+1)} K}
$$


We therefore conclude that

$$
\begin{aligned}
\sum_{i=m}^{\infty} \frac{1}{a_{j}} & >\sum_{k=0}^{2^{m}-1} \frac{1}{a_{m+k}}+\sum_{n=m}^{\infty}\left(\sum_{j=m}^{2^{n}} \frac{1}{a_{2^{n}+j}}\right)>\sum_{n=m}^{\infty}\left(\sum_{j=m}^{2^{n}} \frac{1}{b_{j}}\right) \\
& >\sum_{n=m}^{\infty} \frac{\left(2^{n}-m+1\right)^{2}}{2^{2(n+1)} K}=\frac{1}{K} \sum_{n=m}^{\infty}\left(\frac{1-(m-2) / 2^{n}}{2}\right)^{2} .
\end{aligned}
$$

Since $\frac{1-(m-1) / 2^{n}}{2} \nearrow \frac{1}{2}$ as $n \rightarrow \infty$, we see that

$$
\sum_{i=m}^{\infty} \frac{1}{a_{i}}=\infty
$$

This completes the proof.

We are ready to prove Proposition 5.

Proof of Proposition 5. From condition (A) it follows that

there exists a $K>0$ and a $T_{1}>0$ such that $\int_{0}^{t} h(s) d s<K t^{2}$ for $t \geq T_{1}$.

Since $h$ satisfies condition (3), we see that

(18) there exists a $\nu>0$ and a $T_{2}>0$ such that $\int_{t}^{t+1} h(s) d s \geq \nu$ for $t \geq T_{2}$.

Let $m$ be an integer satisfying $m \geq \max \left\{T_{1}, T_{2}\right\}$. Define

$$
a_{n}=\int_{n-1}^{n} h(s) d s
$$

Then, from (17) and (18), it turns out that $a_{n} \geq \nu>0$ for $n \geq m$ and

$$
\sum_{i=m}^{\ell} a_{i}=\int_{m-1}^{\ell} h(s) d s \leq \int_{0}^{\ell} h(s) d s<K \ell^{2}
$$

for $\ell \geq m$. Hence, by Lemma 6 , condition ( $(\underline{H})$ holds. The proof is complete.

\section{REFERENCES}

[1] Zvi Artstein and E. F. Infante, On the asymptotic stability of oscillators with unbounded damping, Quart. Appl. Math. 34 (1976/77), no. 2, 195-199, DOI 10.1090/qam/466789. MR0466789

[2] Andrea Bacciotti and Lionel Rosier, Liapunov functions and stability in control theory, 2nd ed., Communications and Control Engineering Series, Springer-Verlag, Berlin, 2005. MR2146587

[3] R. J. Ballieu and K. Peiffer, Attractivity of the origin for the equation $\ddot{x}+f(t, x, \dot{x}) \dot{x}^{\alpha} \dot{x}+$ $g(x)=0$, J. Math. Anal. Appl. 65 (1978), no. 2, 321-332, DOI 10.1016/0022-247X(78)90183X. MR506309

[4] Luu Hoang Duc, Achim Ilchmann, Stefan Siegmund, and Peter Taraba, On stability of linear time-varying second-order differential equations, Quart. Appl. Math. 64 (2006), no. 1, 137151, DOI 10.1090/S0033-569X-06-00995-X. MR2211381

[5] A. Halanay, Differential equations: Stability, oscillations, time lags, Academic Press, New York-London, 1966. MR0216103

[6] Jack K. Hale, Ordinary differential equations, 2nd ed., Robert E. Krieger Publishing Co., Inc., Huntington, N.Y., 1980. MR587488 
[7] L. Hatvani, On partial asymptotic stability and instability. III. Energy-like Ljapunov functions, Acta Sci. Math. (Szeged) 49 (1985), no. 1-4, 157-167. MR839935

[8] László Hatvani, On the uniform attractivity of solutions of ordinary differential equations by two Lyapunov functions, Proc. Japan Acad. Ser. A Math. Sci. 67 (1991), no. 5, 162-167. MR.1114963

[9] L. Hatvani, On the asymptotic stability for a two-dimensional linear nonautonomous differential system, Nonlinear Anal. 25 (1995), no. 9-10, 991-1002, DOI 10.1016/0362546X(95)00093-B. MR.1350721

[10] L. Hatvani, Integral conditions on the asymptotic stability for the damped linear oscillator with small damping, Proc. Amer. Math. Soc. 124 (1996), no. 2, 415-422, DOI 10.1090/S00029939-96-03266-2. MR 1317039

[11] László Hatvani, Tibor Krisztin, and Vilmos Totik, A necessary and sufficient condition for the asymptotic stability of the damped oscillator, J. Differential Equations 119 (1995), no. 1, 209-223, DOI 10.1006/jdeq.1995.1087. MR1334491

[12] László Hatvani and Vilmos Totik, Asymptotic stability of the equilibrium of the damped oscillator, Differential Integral Equations 6 (1993), no. 4, 835-848. MR1222304

[13] A. O. Ignatyev, Stability of a linear oscillator with variable parameters, Electron. J. Differential Equations 17 (1997), 1-6. MR1476064

[14] V. M. Matrosov, On the stability of motion, J. Appl. Math. Mech. 26 (1963), 1337-1353, DOI 10.1016/0021-8928(62)90010-2. MR0153934

[15] Anthony N. Michel, Ling Hou, and Derong Liu, Stability of dynamical systems, Systems \& Control: Foundations \& Applications, Birkhäuser Boston, Inc., Boston, MA, 2008. Continuous, discontinuous, and discrete systems. MR2351563

[16] J. J. Levin and J. A. Nohel, Global asymptotic stability for nonlinear systems of differential equations and applications to reactor dynamics, Arch. Rational Mech. Anal. 5 (1960), 194211 (1960), DOI 10.1007/BF00252903. MR0119524

[17] Masakazu Onitsuka and Jitsuro Sugie, Uniform global asymptotic stability for half-linear differential systems with time-varying coefficients, Proc. Roy. Soc. Edinburgh Sect. A 141 (2011), no. 5, 1083-1101, DOI 10.1017/S0308210510000326. MR2838369

[18] Patrizia Pucci and James Serrin, Precise damping conditions for global asymptotic stability for nonlinear second order systems, Acta Math. 170 (1993), no. 2, 275-307, DOI 10.1007/BF02392788. MR 1226530

[19] Patrizia Pucci and James Serrin, Asymptotic stability for intermittently controlled nonlinear oscillators, SIAM J. Math. Anal. 25 (1994), no. 3, 815-835, DOI 10.1137/S0036141092240679. MR 1271312

[20] Nicolas Rouche, P. Habets, and M. Laloy, Stability theory by Liapunov's direct method, Applied Mathematical Sciences, Vol. 22, Springer-Verlag, New York-Heidelberg, 1977. MR0450715

[21] R. A. Smith, Asymptotic stability of $x^{\prime \prime}+a(t) x^{\prime}+x=0$, Quart. J. Math. Oxford Ser. (2) 12 (1961), 123-126, DOI 10.1093/qmath/12.1.123. MR0124582

[22] Aaron Strauss and James A. Yorke, Perturbing uniform asymptotically stable nonlinear systems, J. Differential Equations 6 (1969), 452-483, DOI 10.1016/0022-0396(69)90004-7. MR.0252781

[23] Aaron Strauss and James A. Yorke, Perturbing uniformly stable linear systems with and without attraction, SIAM J. Appl. Math. 17 (1969), 725-738, DOI 10.1137/0117066. MR0252782

[24] Jitsuro Sugie, Convergence of solutions of time-varying linear systems with integrable forcing term, Bull. Aust. Math. Soc. 78 (2008), no. 3, 445-462, DOI 10.1017/S000497270800083X. MR2472280

[25] Jitsuro Sugie, Influence of anti-diagonals on the asymptotic stability for linear differential systems, Monatsh. Math. 157 (2009), no. 2, 163-176, DOI 10.1007/s00605-008-0030-x. MR2504784

[26] Jitsuro Sugie, Global asymptotic stability for damped half-linear oscillators, Nonlinear Anal. 74 (2011), no. 18, 7151-7167, DOI 10.1016/j.na.2011.07.028. MR2833701

[27] Jitsuro Sugie, Saori Hata, and Masakazu Onitsuka, Global attractivity for half-linear differential systems with periodic coefficients, J. Math. Anal. Appl. 371 (2010), no. 1, 95-112, DOI 10.1016/j.jmaa.2010.04.035. MR2660989 
[28] Jitsuro Sugie and Masakazu Onitsuka, Global asymptotic stability for half-linear differential systems with coefficients of indefinite sign, Arch. Math. (Brno) 44 (2008), no. 4, 317-334. MR.2493428

[29] Jitsuro Sugie and Masakazu Onitsuka, Integral conditions on the uniform asymptotic stability for two-dimensional linear systems with time-varying coefficients, Proc. Amer. Math. Soc. 138 (2010), no. 7, 2493-2503, DOI 10.1090/S0002-9939-10-10274-3. MR2607879

[30] Jitsuro Sugie and Masakazu Onitsuka, Growth conditions for uniform asymptotic stability of damped oscillators, Nonlinear Anal. 98 (2014), 83-103, DOI 10.1016/j.na.2013.12.005. MR.3158447

[31] A. G. Surkov, Asymptotic stability of certain two-dimensional linear systems (Russian), Differentsial'nye Uravneniya 20 (1984), no. 8, 1452-1454. MR759607

[32] Taro Yoshizawa, Stability theory by Liapunov's second method, Publications of the Mathematical Society of Japan, No. 9, The Mathematical Society of Japan, Tokyo, 1966. MR.0208086

Department of Mathematics, Shimane University, Matsue 690-8504, Japan

E-mail address: kazu2520asyst@gmail.com

Department of Mathematics, Shimane University, Matsue 690-8504, Japan

E-mail address: jsugie@riko.shimane-u.ac.jp 